\title{
A review on the COVID-19-related psychological impact on older adults: vulnerable or not?
}

\author{
Eleni Parlapani ${ }^{1}\left[\right.$ - Vasiliki Holeva ${ }^{1} \cdot$ Vasiliki Aliki Nikopoulou ${ }^{1} \cdot$ Stergios Kaprinis $^{2} \cdot$ Ioannis Nouskas $^{3}$. \\ loannis Diakogiannis ${ }^{1}$
}

Received: 7 February 2021 / Accepted: 28 April 2021 / Published online: 17 May 2021

(c) The Author(s), under exclusive licence to Springer Nature Switzerland AG 2021

\begin{abstract}
Background COVID-19 is a novel event of the twenty-first century. Therefore, contemporary research is required to determine the current pandemic's psychological impact on older populations. Soon after the COVID-19 outbreak, several narrative reviews and guidelines were released to support older adult's psychological wellbeing. However, a lack of data from original studies was reported until May 2020.

Aim To identify studies published from May 2020 until January 2021 that quantitatively assessed the mental health impact of COVID-19 on older adults using validated psychometric tools.

Method A comprehensive literature search of original research articles was conducted using specific terms. The screening procedure was conducted stepwise.

Results Among the 18 included studies, cross-sectional online surveys indicated that older adults were less psychologically distressed than younger ones. A longitudinal study revealed that COVID-19 did not have a major impact on loneliness and satisfaction with life. On the contrary, studies comparing pre- with peri-pandemic data revealed that older adults experienced more severe depressive/anxiety/stress symptoms and loneliness during the pandemic. Several studies reported though only subclinical symptoms, as well as low loneliness levels during the pandemic.

Conclusions Research studies suggested that older age may buffer against the COVID-19-related impact on mental health. Despite evidence against generalised perceptions of vulnerability, older adults' ability to adapt to adversity may be uneven, depending on cultural, social, economic and other individual factors. Taken together, the impact, moreover the long-term impact of COVID-19, is expected to vary across countries and older subpopulations, and remains to be evaluated by prospective, longitudinal studies.
\end{abstract}

Keywords COVID-19 $\cdot$ Older adults $\cdot$ Depressive symptoms $\cdot$ Anxiety symptoms $\cdot$ Post-traumatic stress $\cdot$ Loneliness

Eleni Parlapani

eparlapa@auth.gr

1 Department of Psychiatry, School of Medicine, Faculty of Health Sciences, Aristotle University of Thessaloniki, General Hospital "Papageorgiou”, Ring Road Thessaloniki, N. Efkarpia, 54603 Thessaloniki, Greece

2 Department of Psychiatry, School of Medicine, Faculty of Health Sciences, Aristotle University of Thessaloniki, Thessaloniki, Greece

3 AFFIDEA Center of Medical Diagnosis, Thessaloniki, Greece

\section{Introduction}

The last most severe pandemic in recent history, the 1918 influenza pandemic or "Spanish flu", began in March 1918 and spread worldwide during the following two years. It was estimated that about 500 million people were infected by the H1N1 influenza A virus. Owing to a lack of vaccine and shortage of antibiotics against secondary bacterial infections, the pandemic caused the death of at least 50 million people worldwide. The efforts to prevent the spread of the virus were focused on standard precautions, that is, personal hygiene and use of face masks. Nationwide restriction measures, including quarantine, prohibition of public gathering and restriction of movement, were also applied in countries worldwide, although unevenly [1]. A century later, the world 
is reliving a pandemic, caused by the novel SARS-CoV-2 virus. To date, the virus infected around 100 million people, causing the death of over 2 million people worldwide [2]. Although the statistics on the current pandemic may not be comparable to those on the 1918 influenza pandemic, COVID-19 is undoubtably a severe infectious disease outbreak, considering the advances in medicine and the availability of medical technology during the twenty-first century. For the first time after 100 years, restriction measures and national lockdowns are again imposed worldwide to reduce the spread of a virus.

Soon after the COVID-19 outbreak, diverse public health institutes, organisations and stakeholders highlighted the importance of fostering older adults' physical health and mental wellbeing. Older adults are of particular concern on two grounds. First, they comprise a steadily growing demographic subgroup. In 2019, older adults over the age of 65 accounted for $20.3 \%$ of the European Union population, an increase of $0.3 \%$ when compared with the previous year and of $2.9 \%$ compared with 10 years before. Moreover, the subpopulation of the older old (over the age of 80 ) is expected to increase from $5.8 \%$ in 2019 to $14.6 \%$ in 2100 . In 2019, the old-age dependency ratio (OADR) in Europe was estimated at $31.4 \%$, that is, there were around three working age people for every person over the age of 65 [3]. Altogether, countries all over the world are experiencing a longevity revolution to a greater or lesser degree. In contrast to the data from 2019 , indicating that one out of 11 adults exceeded the age of 65 , the age of 65 will be exceeded by one out of six adults worldwide in 30 years from now. In certain regions, such as in Northern Africa, Western-Central-Southern Asia and Latin America, the proportion of older adults is expected to double in the next 30 years. Moreover, an increased life expectancy has been recorded worldwide; in 2015-2020, life expectancy over the age of 65 was globally estimated at 17 years, whereas in 2045-2050, older adults are expected to reach the age of 84 ; in most developed countries, the proportion of older adult life will correspond to one quarter of total life time [4].

Secondly, advanced age is related with intrinsic and extrinsic factors posing greater risks for older adults during public emergency crises [5]. As such, several narrative reports outlined the risks and challenges for older adults posed directly and indirectly after the COVID-19 outbreak $[6,7]$ :

(1) Advanced age was timely identified as a risk factor for severe disease and increased mortality, possibly due to the age-related immunosenescence [8]. In addition, the higher prevalence of chronic conditions in older adults [9], as well as health conditions related with obesity [10], pose a higher risk for severe COVID-19. Based on the data obtained from 19 countries during a six- week period in April-May 2020, 86.2\% of COVID19-related deaths were reported in patients over the age of 65 ; mortality rates were 8.1 times higher in individuals aged 55-64 years, whereas adults over the age of 65 showed 7.7 times higher death rates than those aged 55-64 years [11]. Consequently, older age may be related with more fear of COVID-19 [12] and greater COVID-19 worry [13];

(2) The risk of discrimination in COVID-19 patient care due to medical equipment shortages during the pandemic may have been greater for older adults, despite efforts to prevent inequity in healthcare provision [14]. At the first COVID-19 wave outbreak, younger patients were prioritised over older, particularly in countries severely hit by the pandemic, where the healthcare system was overloaded with COVID-19 patients [15]. In addition, a systematic review addressed the underrepresentation of older adults in randomised controlled trials on the pharmacologic treatment of COVID-19 [16];

(3) As a result of the higher prevalence of chronic diseases, older people require regular doctor visits and long-term medication. Therefore, suspension and postponement of health services for nonemergent conditions unrelated to COVID-19 probably had a greater impact on older patients [17]. In addition, fear of contracting the virus may have been associated with decreased hospital visits and hospitalisations for other conditions. Despite lack of conclusive data, anecdotal records suggested markedly decreased visits in various departments [18];

(4) Older adults living under overcrowded conditions may have been particularly endangered, such as refugees in camps (due to limited visits by members of nongovernmental organisations), older people in unauthorised housing (due to limited access to charity aid), as well as older prisoners [17];

(5) During the quarantine, isolation and physical distancing may have had a greater impact on dependent older old people living alone and in need of home care. Fear of infection limited care to provision of basic needs, disrupting regular home visits. Therefore, physically and mentally vulnerable older adults were at higher risk of neglect [18];

(6) Long-period quarantine together with family members, cancellation of regular appointments, due to closure of senior centres and limited access to support services may have increased the danger of physical/emotional abuse and/or financial exploitation of older adults [19];

(7) Contained acquaintance with the internet, as well as limited familiarity with technology overall, e.g. social media, video calling, may have intensified the impact of social isolation on older adults. Stay-at-home orders may have deprived them from companionship and the 
sense of connectedness, intensifying feelings of loneliness and reducing wellbeing [20];

(8) COVID-19 has affected both life and death. COVID-19 victims die isolated from their loved ones. Moreover, the pandemic crisis has not enabled mourning and grief in the comfort of others. Carried away with the pandemic, one should not forget that COVID-19 is not the only disease; older patients suffering from other severe or deadly diseases have also been isolated from their significant others [21].

Presuming that the threat imposed by the virus and the quarantine-related social isolation would endanger older adults' psychological wellbeing, mental health professionals contributed to the release of narrative reviews, theoretical reports and guidelines to support older people. Articles focused, among others, on psychological factors and behavioural patterns that may help older adults shield themselves from negative emotions [22], highlighted the sense of "mattering" and its protective role against mental health problems [23], summarised the pandemic's impact on the management of noncommunicable diseases in older Europeans, recommended approaches to promote healthy ageing [24], and addressed the needs of older patients with pre-existing mental disorders [25]. Other narrative reviews presented smartphone apps and remotely delivered interventions to address social isolation [26, 27], and proposed measures against older adult's marginalisation [6].

Despite the great amount of literature on the vulnerability of older adults, many reports were narrative and based on previous experience with medical health crises and natural disasters. The outbreak of COVID-19 evoked an alert response of fear, related with the biodemographic burden and behavioural impact of past infectious disease outbreaks [28]. However, COVID-19 is a novel event of the twentyfirst century. People worldwide experience the benefits of advances in healthcare provision, medical technology and pharmaceuticals. Immediately after the COVID-19 outbreak, researchers all over the world timely responded to the crisis providing epidemiological data and research findings related with prevention and treatment strategies. Moreover, innovative digital technologies have been developed for the containment of COVID-19, among which genomics, digital diagnostics and smartphone apps [29]. In addition, the pandemic boosted the technological progress in communication. During the lockdown, older adults were proven to be adaptable, since they increased use of technology to remotely maintain social connections, an approach related with better mental health outcomes [30]. Therefore, contemporary research is required to determine the current pandemic's psychological impact on older populations.

An early rapid review focusing on the mental health sequelae of the pandemic was released in May 2020.
Although the study raised awareness about the potential vulnerability of older adults towards mental distress, the authors pointed out the lack of data from original research articles [31]. Similarly, a review released in June, 2020, outlined the lack of data on older patients and highlighted the need and importance of more focused research on the COVID19-related impact on older adults [32].

Considering the novelty of COVID-19, the vulnerability in older age and the reported limited research on older people until May 2020, a comprehensive search of original studies of older individuals was conducted. The aim was: (i) to identify studies published from May 2020 until January 2021 that quantitatively assessed the mental health impact of COVID-19 on older adults using validated psychometric tools; (ii) to summarise data and (iii) to collectively draw conclusions about the magnitude of older adult's psychological distress, caused by the pandemic and the quarantinerelated measures.

\section{Search strategy and selection criteria}

A literature search was conducted on PubMed on Jan 17, 2020, using the following search string: "COVID-19" OR "Sars-Cov-2" AND "mental health" AND "elderly" OR "geriatric" OR "older". During the search, the following filters were applied: (i) journal article; (ii) publication date from 2020/5/1 to 2021/1/17; (iii) human studies and (iv) articles in English. The screening procedure was conducted stepwise.

At the first step, records were screened for their title and abstract. The selection criterion was articles addressing mental health issues of older adults and/or reporting an age effect on the psychological impact of COVID-19. All types of review articles, studies of other specified populations (e.g. healthcare professionals, physical therapists, caregivers of COVID-19 survivors, women in maternity, psychiatric patients, Parkinson patients, cancer patients etc.), studies without focus on older populations and studies of older adults defined as "patients" (older patients with COVID-19, pre-existing psychiatric disorders or neurocognitive disorders, e.g., mild cognitive impairment, Alzheimer's disease) were excluded.

At the second step, the full-text of the remaining records was evaluated to retrieve articles that fulfilled the review's inclusion criteria: (i) original research articles; (ii) focus on the impact of COVID-19 on mental health; (iii) data obtained from samples with a mean age over the age of 60 ; (iv) use of validated psychometric tools to assess the psychological burden, that is, depressive, anxiety, stress and posttraumatic stress symptoms, insomnia, loneliness and quality of life and (v) reported mean (SD) values and/or prevalence rates (\%). There were no restrictions with regard to study design, that is, cross-sectional or longitudinal, online survey 
or in-person assessment. Exclusion criteria were: (i) theoretical, narrative and recommendation reports, commentaries, editorials, letters to the editor, case reports/series and reviews; (ii) qualitative studies; (iii) study protocols; (iv) intervention studies; (v) scale validation studies.

\section{Results}

\section{Characteristics of included studies}

A total of 8771 records were retrieved. At the first screening step, 133 records were selected based on title/abstract relevance with the review's scope. Among these, 18 fulfilled inclusion criteria at the second screening step (Fig. 1). Among the included studies, 10 were cross-sectional online surveys during the acute phase of the pandemic [33-42], five studies compared data collected prior with the data collected during the pandemic [43-47], two studies reported baseline data from ongoing longitudinal studies [48, 49] and one study reported longitudinal data [50] (Table 1).

\section{Depression, anxiety, stress and insomnia}

A cross-sectional study, conducted through an online survey in Cyprus two weeks after the national lockdown, reported that older participants showed significantly lower mean Patient Health Questionnaire depression scale (PHQ-9) score $(M=3.90, \mathrm{SD}=3.57)$ and mean Generalised Anxiety Disorder scale (GAD-7) score $(M=4.83, \mathrm{SD}=4.22)$, when compared with participants aged 18-29 years $\left(M_{\mathrm{PHQ}-9}=8.47, \mathrm{SD}=5.79 ; M_{\mathrm{GAD}-7}=7.61, \mathrm{SD}=4.93\right)$ [33]. Similarly, an one-day online survey on subscribers enrolled in the Text4Hope, a program developed to support Albertans during the COVID-19 pandemic, revealed that adults over the age of 60 showed significantly lower mean PHQ-9 and GAD-7 scores, when compared with younger age groups; the greatest mean differences were observed between respondents younger than 25 and older than 60 years [34].

Fig. 1 Flow chart of the search strategy

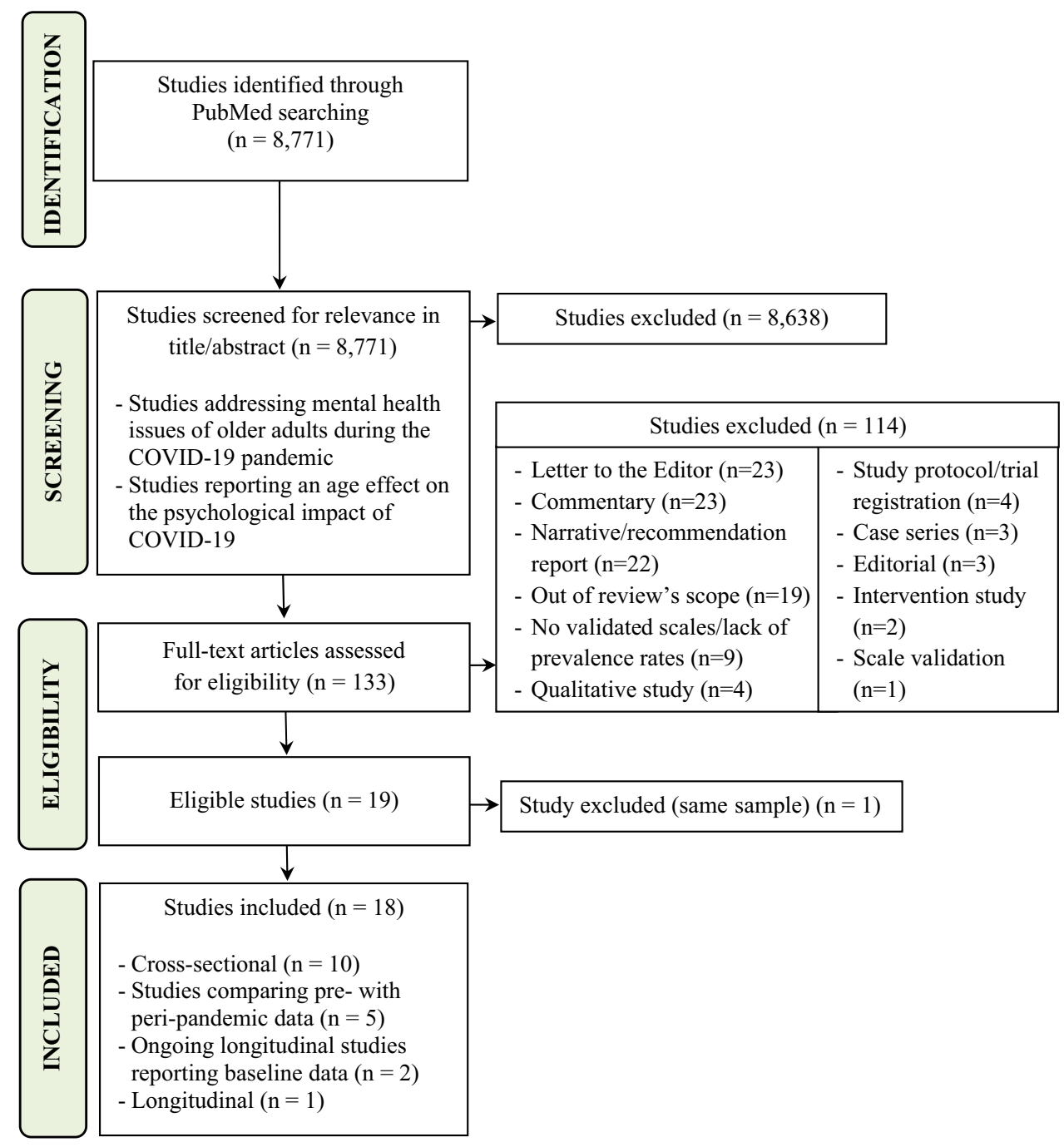




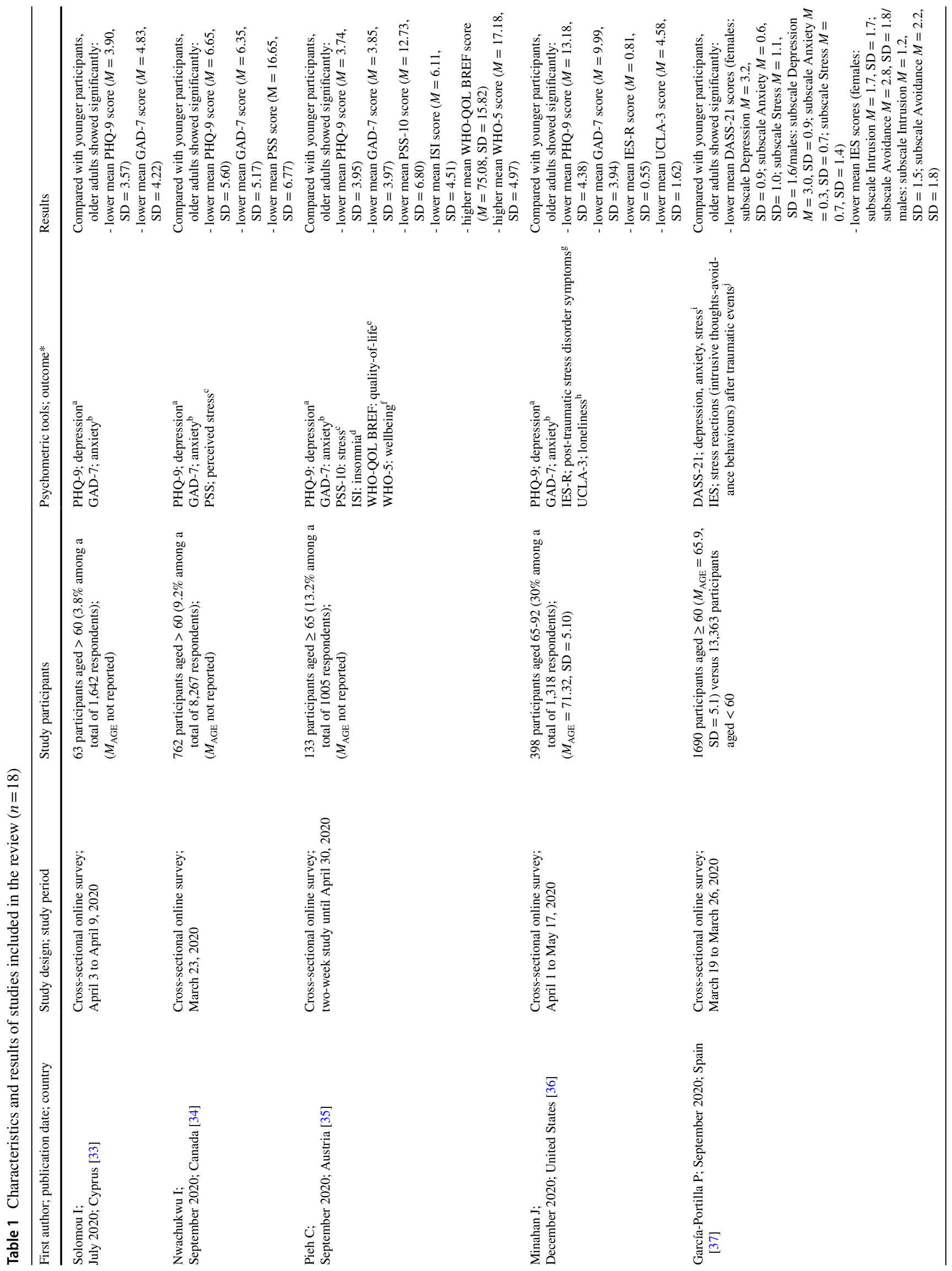




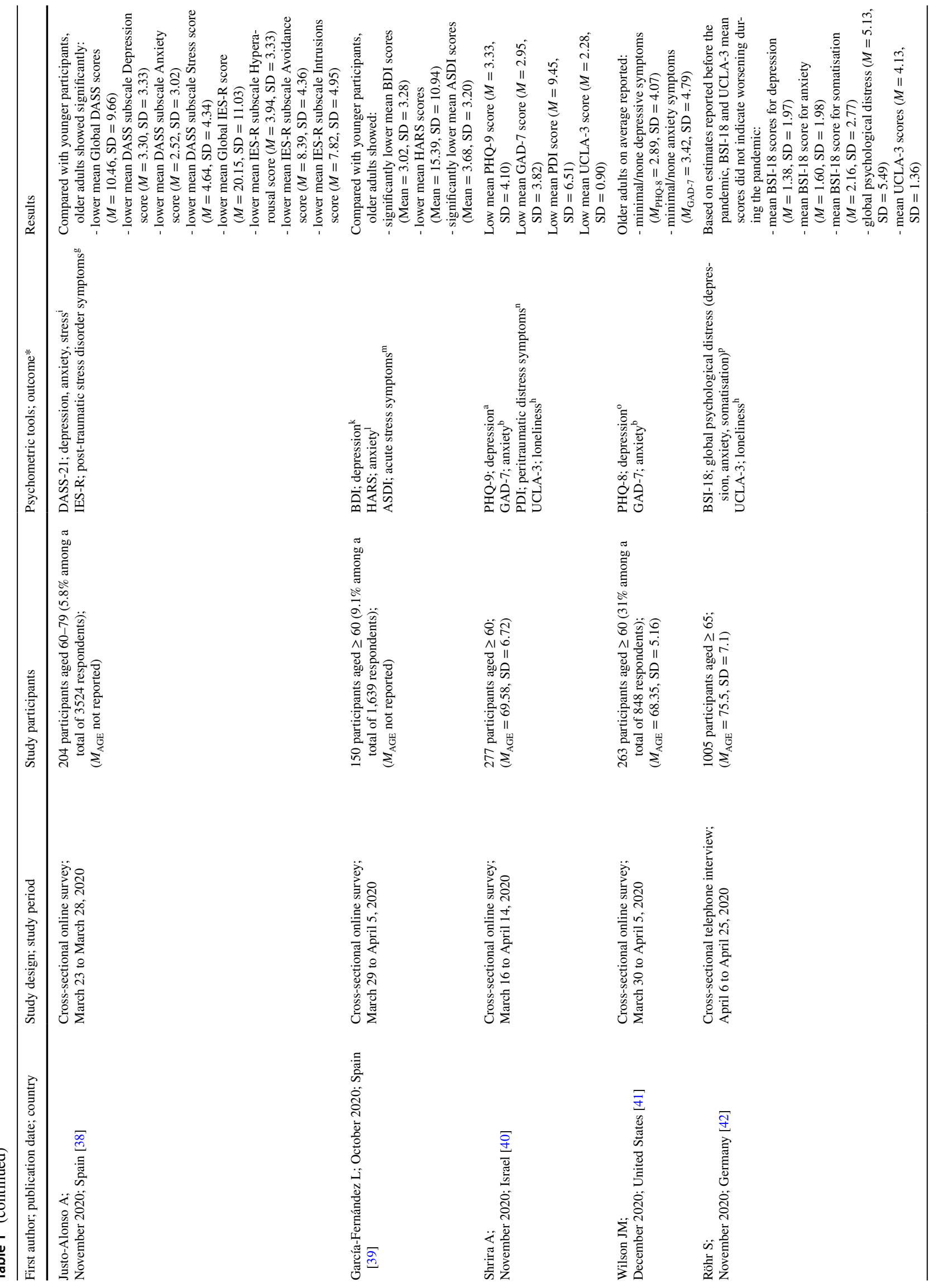




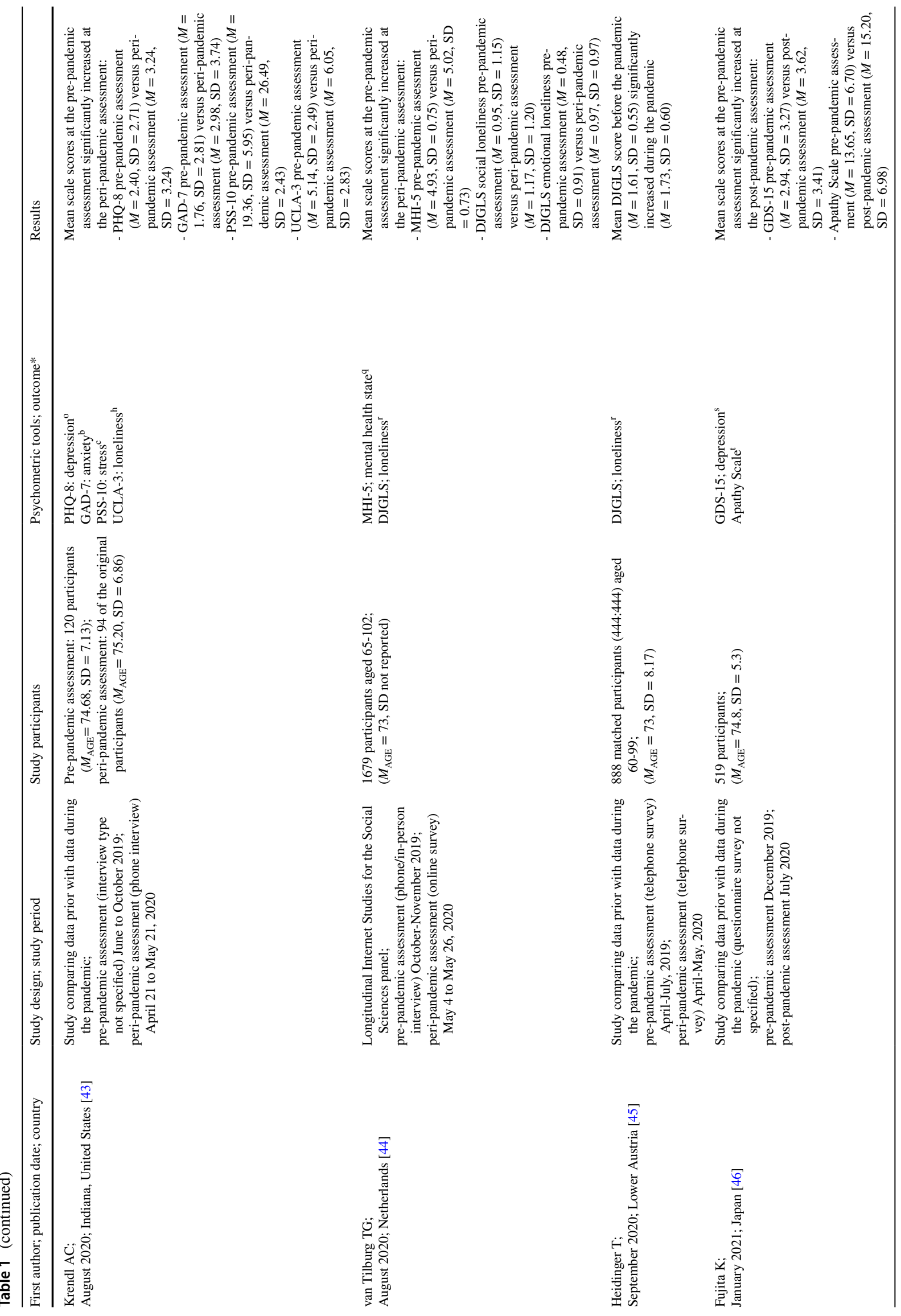




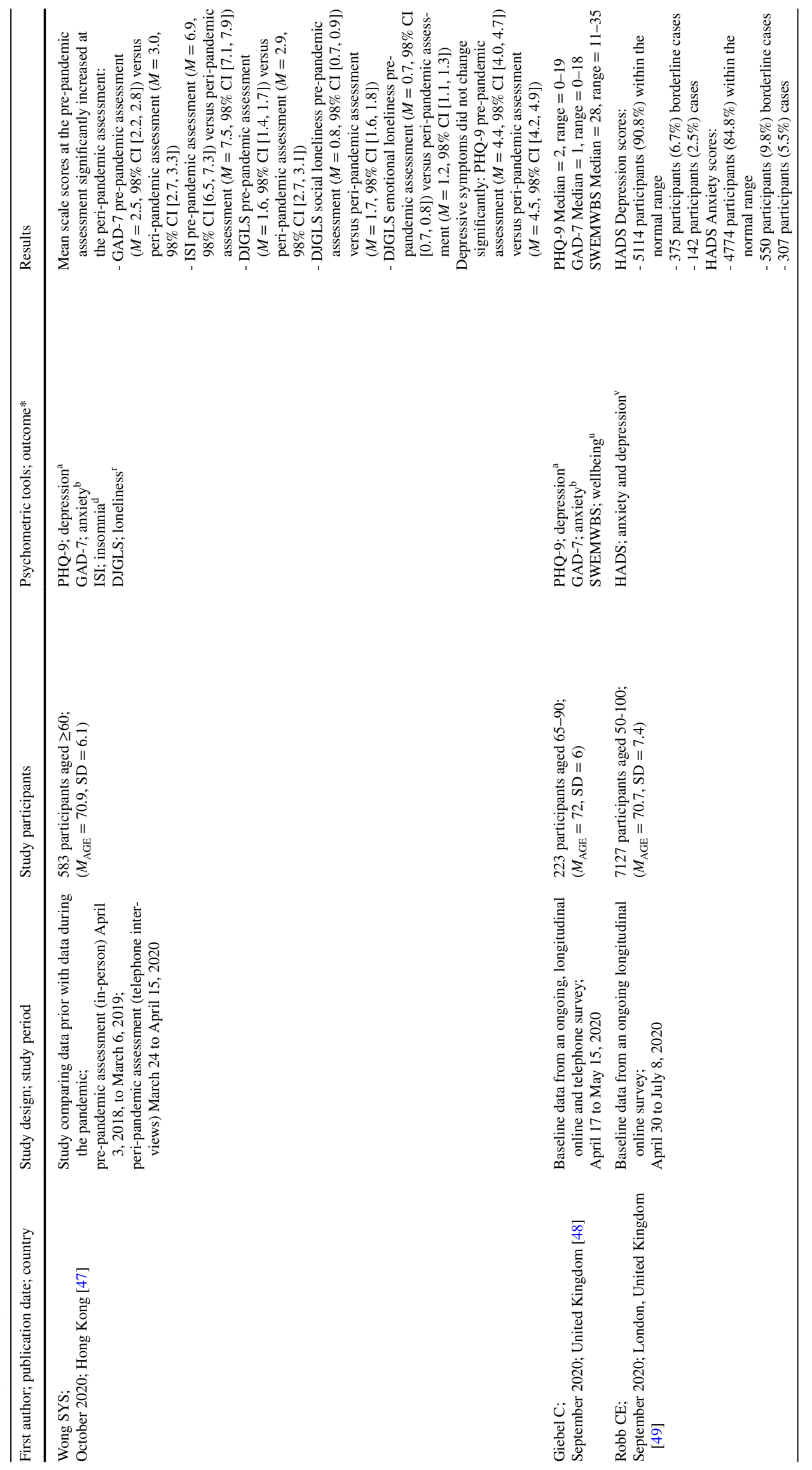




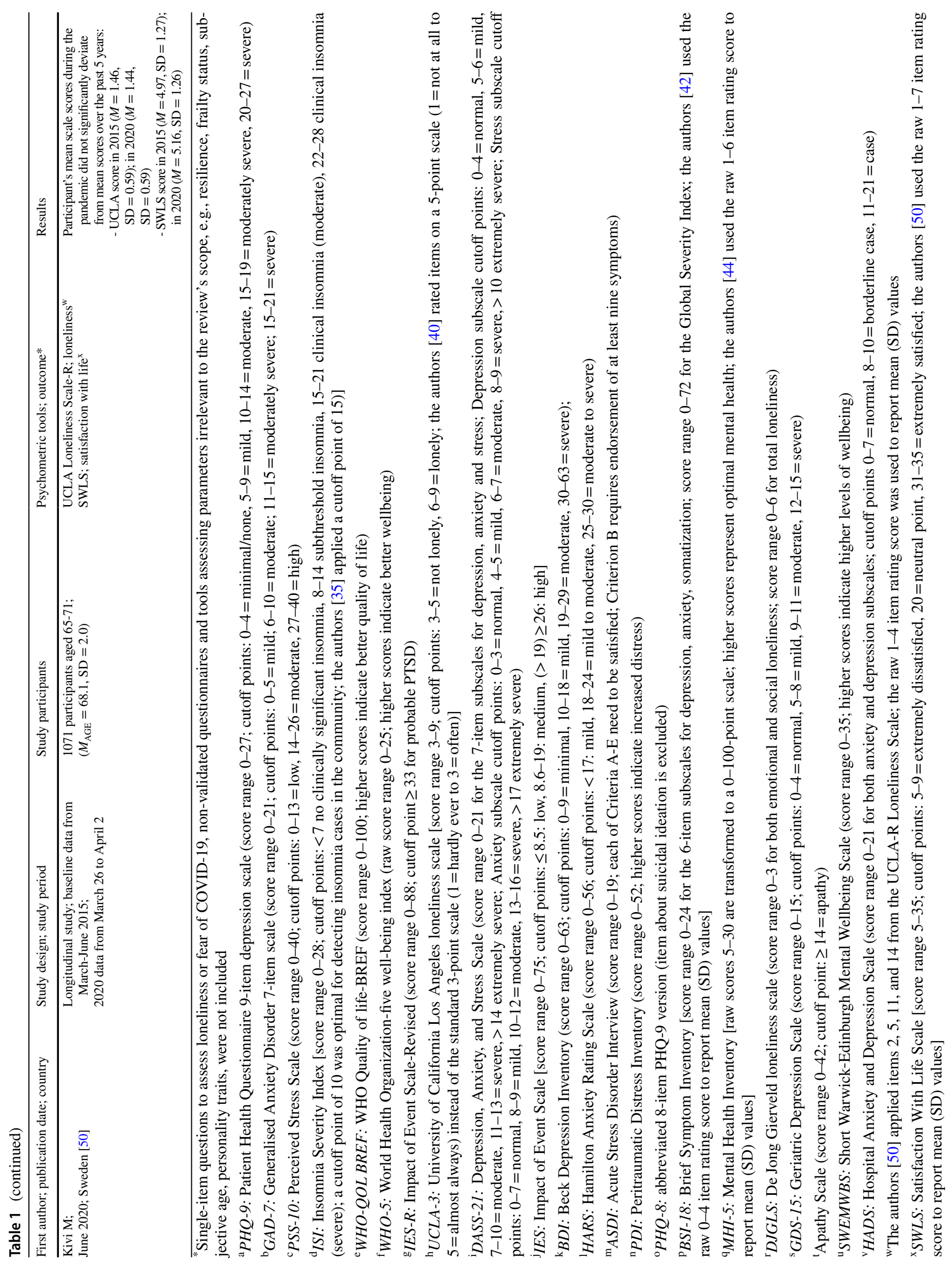


Accordingly, a two-week online survey, conducted during the four-week quarantine in Austria, showed again lower mean PHQ-9 and GAD-7 scores in older, compared with younger participants; the prevalence of clinically significant depressive and anxiety symptoms in adults over the age of 65 was low (5.3\%) [35]. Less severe depressive and anxiety symptoms in older than in younger adults, indicated by lower mean PHQ-9 and GAD-7 scores, were confirmed by an online survey conducted during the initial quarantine in the United States [36].

Three Spanish online surveys, conducted during the quarantine, used a snowball sampling technique. The first reported that older female and male participant's mean Depression, Anxiety and Stress Scale (DASS-21) score was significantly lower than mean scores observed in female and male participants younger than the age of 60 [37]; the second confirmed the previous report, since participants over the age of 60 displayed lower mean DASS-21 score compared with all younger age groups [38]; the third reported that the difference between older participants' mean Beck Depression Inventory (BDI) score $(M=3.02, \mathrm{SD}=3.28)$ and younger participant's mean BDI score $(M=4.30, \mathrm{SD}=4.93)$ was statistically significant; although participants over the age of 60 displayed lower mean Hamilton Anxiety Rating Scale (HARS) scores than younger ones, the difference was not significant (healthcare professionals and participants with past/current history of mental illness were excluded from the analysis) [39].

Low levels of anxiety and depressive symptoms were again reported by an online survey using convenience sampling in Israel (low mean PHQ-9 and GAD-7 scores) [40], as well as by an online survey of a national representative sample in the United States, stating that the majority of older people (over 70\%) reported none to minimal depressive and anxiety symptoms [41]. Lastly, according to a crosssectional, computer-assisted telephone interview conducted during the national lockdown in Germany (random selection of a representative sample of adults over the age of 65), mean Brief Symptom Inventory (BSI-18) score for depression, anxiety, somatisation and global psychological distress did not indicate worse mental wellbeing during the COVID-19 lockdown, based on normative values and estimates reported before the pandemic [42].

Older Albertans showed significantly lower mean Perceived Stress Scale (PSS-10) score than younger ones; the greatest mean differences were again observed between respondents younger than 25 and older than 60 years [34]. Similarly, older Austrians showed lower mean PSS-10 score, compared with younger ones, as well as lower mean Insomnia Severity Index (ISI) score; the prevalence of insomnia was low $(6.8 \%)$ [35].
Three studies compared data before with the data during the pandemic, to assess the psychological impact of COVID19. The first one, conducted in the United States, compared data collected in June-October, 2019 (data collection method not reported), to assess the impact of older adults' social relationships on overall wellbeing, with the data collected from the same sample through phone interview around a month after the shelter-in-place order had been issued in Bloomington, Indiana. According to the results, reported only for the 87 participants still sheltering-in-place at the time of data collection, older adults experienced more severe depressive, anxiety and stress symptoms (assessed by PHQ-8, GAD-7 and PSS-10 respectively) during the pandemic, compared with prior to the pandemic [43]. The second compared data obtained from older adults participating in the TENG project (Toyoyama town complex intervention project promoting Exercise, Nutrition improvement and Going out; questionnaire survey) at two different time points, in December 2019 (pre-pandemic) and in July 2020 (post-pandemic). Both depressive symptoms and levels of apathy increased at the post-pandemic assessment. Subgroup analyses revealed that both mean Geriatric Depression Scale (GDS-15) and Apathy Scale scores significantly increased in those younger than 75 years of age (Mean raw GDS-15 score increase $=0.87, \mathrm{SD}=3.31$; Mean raw Apathy Scale score increase $=2.17, \mathrm{SD}=6.28$ ), whereas in those over the age of 75, only the GDS-15 score significantly increased (Mean raw GDS-15 score increase $=0.57, \mathrm{SD}=3.57$ ) [46]. Lastly, a study reported data from a telephone survey on a pre-existing cohort of adults over the age of 60 with multimorbidity (more than two chronic conditions) in primary care in Hong Kong. The first assessment was conducted in-person before the pandemic, whereas the second through telephone interviews during the pandemic. According to the results, depressive symptoms' severity did not change, whereas the severity of anxiety symptoms and insomnia significantly increased during the pandemic [47].

Contrary to the previous reports, mental health assessed by the Mental Health Inventory (MHI-5) was shown to significantly improve during the pandemic. This outcome was reported by a study comparing data from a random sample of the Longitudinal Internet Studies for the Social Sciences (LISS) panel in the Netherlands (data collected in October-November 2019; participants were recruited by letter, followed by a telephone call or house visit), with the data from 1679 members of the longitudinal panel answering online questions about the social impact of physical distancing (data collected two months after the implementation of measures in the Netherlands) [44].

Lastly, two studies reported baseline data from ongoing longitudinal studies in the United Kingdom. The first one, an ongoing online and telephone survey, reported that only a few older adults showed clinically significant depressive 
and anxiety symptoms (5\%), assessed by PHQ-9 and GAD7, respectively [48]. The second was the baseline survey of the ongoing CHARIOT COVID-19 Rapid Response Study (CCRR), conducted within six weeks of lockdown in London. Participants were contacted via email or post and completed an online survey. The majority (90.8\%) did not report clinically significant depressive symptoms, whereas only $5.5 \%$ reported clinically significant anxiety symptoms [49].

\section{Post-traumatic stress symptoms}

An online survey during the initial quarantine in the United States reported that adults aged 65-92 years displayed less severe post-traumatic stress symptoms, indicated by the lower mean Impact of Event Scale-Revised (IES-R) score $(M=0.81, \mathrm{SD}=0.55)$, compared with the mean IES-R scores observed in adults aged $40-64(M=0.97, \mathrm{SD}=0.61)$ and 18-39 years $(M=1.19, \mathrm{SD}=0.67)[36]$.

Similarly, three online surveys conducted during the national lockdown in Spain revealed that older adults displayed less severe post-traumatic stress symptoms compare with younger ones. The first one reported that Spaniards over the age of 60 , both female and male, showed significantly lower mean Impact of Event Scale (IES) score, compared with those younger than the age of 60 [37]. The second confirmed the previous findings, since adults over the age of 60 displayed significantly lower mean IES-R score $(M=20.15$, $\mathrm{SD}=11.03)$ compared with all younger age groups [46-60 years $(M=21.85, \mathrm{SD}=12) ; 34-45$ years $(M=24.22$, $\mathrm{SD}=11.99) ; 26-33$ years $(\mathrm{M}=25.17, \mathrm{SD}=12.23) ; 18-25$ years $(M=25.33, S D=11.77)$ ] [38]. In accordance, the third study reported that adults over the age of 60 showed significantly lower mean Acute Stress Disorder Inventory (ASDI) score $(M=3.68, \mathrm{SD}=3.20)$, compared with younger adults $(M=4.45, \mathrm{SD}=3.06)$ [39]. Lastly, a cross-sectional online survey conducted in Israel reported low levels of peritraumatic distress symptoms in older adults, indicated by the low mean Peritraumatic Distress Inventory (PDI) score [40].

\section{Loneliness}

An online survey conducted during the initial quarantine in the United States revealed that adults over the age of 65 reported lower mean University of California Los Angeles Loneliness Scale-3 items (UCLA-3) score ( $M=4.58$, $\mathrm{SD}=1.62)$, compared with younger participants [40-64 years $(M=5.17, \mathrm{SD}=1.86) ; 18-39$ years $(M=5.56(1.73)$ ] [36]. In accordance, an online survey in Israel reported that older adults showed low levels of loneliness, indicated by the low mean UCLA-3 score [40]. Similarly, a German crosssectional study reported that mean UCLA-3 scores and loneliness prevalence (13.1\%) did not indicate an increase in loneliness during the COVID-19 lockdown, based on estimates reported before the pandemic [42].

In contrast to the previous reports, a study comparing prewith peri-pandemic data reported that older Indiana residents still sheltering-in-place experienced higher levels of loneliness, assessed by UCLA-3, during the pandemic [43]. Similarly, another study comparing pre-with peri-pandemic data reported that older adults' social and emotional loneliness levels, assessed by the De Jong Gierveld Loneliness Scale (DJGLS), increased during the pandemic in the Netherlands [44]. A slight, but significant increase in loneliness levels was also observed in older residents of Lower Austria, according to a study that compared data from two standardised, representative community-based telephone surveys, a pre-pandemic (April-July, 2019; $N=2042$ ) and a peri-pandemic (April-, 2020; $N=521$ ). The matched sample consisted of 888 adults (444:444), aged 60-99 years. Despite the increase in loneliness levels during the pandemic, the majority of the matched participants (75\%) showed low loneliness scores $\left(M_{\mathrm{DJGLS}}=1.67, \mathrm{SD}=0.58\right)$; less than $3 \%$ showed high/very high loneliness levels [45]. Lastly, older adults with multimorbidity in primary care in Hong Kong also experienced more loneliness during the pandemic. Before COVID-19, 59.5\% of the participants were not lonely, while $8.8 \%$ were severely lonely; during the pandemic, the proportion of not lonely adults decreased (29.9\%), whereas the proportion of severely lonely adults increased (27.7\%) [47].

The only longitudinal study included in this review reported data from a subsample of the HEalth, Ageing and Retirement Transitions in Sweden (HEARTS) study, that is, an annual survey in a population-based sample of adults born 1949-1955. The baseline data were collected in March-June 2015, whereas 2020 data were collected from participants responding during the COVID-19 pandemic. During the 2020 7-day study period, Swedish authorities had recommended social distancing, as well as "shelter-in-place" for adults over the age of 70, without imposing a national lockdown. According to the results, loneliness levels did not change significantly during the COVID-19 pandemic [50].

\section{Quality of life, wellbeing and satisfaction with life}

An online survey conducted during the lockdown in Austria revealed that adults over the age of 65 showed higher levels of quality of life, assessed by the WHO Quality of lifeBREF (WHO-QOL BREF; $M=75.08, \mathrm{SD}=15.82$ ), as well as better wellbeing, assessed by the WHO-Five Well-Being Index (WHO-5; $M=17.18, \mathrm{SD}=4.97$ ), compared with younger participants of different age groups; adults aged 25-34 and 18-24 years showed the lowest levels of quality of life and well-being [25-34 years $\left(M_{\mathrm{WHO}-\mathrm{QOLBREF}}=67.35\right.$, $\left.\mathrm{SD}=19.12 ; M_{\mathrm{WHO}-5}=14.20, \mathrm{SD}=5.26\right) ; 18-24$ years 
$\left(\mathrm{M}_{\mathrm{WHO}-\mathrm{QOLBREF}}=65.72, \mathrm{SD}=20.32 ; M_{\mathrm{WHO}-5}=13.63\right.$, $\mathrm{SD}=4.91)$ ] [35]. Similarly, adults over the age of 65 in the United Kingdom displayed relative high levels of wellbeing, assessed by the Short Warwick-Edinburgh Mental Wellbeing Scale (SWEMWBS) [48].

Lastly, according to the HEARTS longitudinal study, older adults' satisfaction with life, assessed by the Satisfaction With Life Scale (SWLS), did not change significantly during the COVID-19 in Sweden [50].

\section{Discussion}

Research during the pandemic indicated that although older adults may express more pronounced fear of COVID-19, possibly related with the mass media announcements about their higher risk for severe illness and increased COVID19-related mortality, generalised anxiety symptoms may decrease with age [51]. In addition, older age was related with less depressive symptoms [52] and psychological distress [53]. Furthermore, there was no evidence that older old adults (71-80 years) show a poorer psychological wellbeing during the pandemic, compared with younger old adults [54].

Considering the above, as well as the reports of limited research on older people until May 2020 [31, 32], this review aimed to identify original research studies of older populations published in the last nine months and to discuss the existing data on the COVID-19-related psychological impact on older adults.

The majority of the studies included in this review were of cross-sectional design. Pandemics elicit alert responses of fear and anxiety, related with collective memories of the biodemographic burden of past infectious disease outbreaks [28]. Therefore, the interpretation of the data from crosssectional studies during the acute phase of the pandemic is limited, unless normative values and estimates reported before the pandemic are available [42].

Owing to the strict quarantine measures, most crosssectional studies were conducted through online surveys. Although there is evidence that online data may be equivalent to paper-and-pencil data $[55,56]$, data quality of online surveys using self-administered questionnaires continues to receive criticism. Furthermore, individuals may particularly respond to online surveys when the survey's scope is relevant or important to them. Consequently, respondents' characteristics may substantially deviate from nonrespondents. Altogether, online surveys may suffer from a volunteer effect and bias related with self-selection [57].

Most importantly, the internet community may not be representative of the older general population, since older adults over the age of 80 are less likely to use the internet; lower educational background, lower socioeconomic status, as well as restrictions due to health issues are factors that limit older adult's internet access. In addition, internet use varies between different countries, e.g., the rates of older adults online are higher in the North-West (50-83\%) than in the East-South of Europe (27-39\%) [58]. Consequently, older adults were underrepresented in some online surveys, comprising $1.7 \%$ [38], 3.8\% [33], 9.15\% [39], 9.2\% [34] or $13.2 \%$ of the total sample [35].

Taking these limitations into consideration, cross-sectional studies comparing older with younger adults indicated that older adults over the age of 60 reported the least severe depressive and anxiety symptoms [33, 41]. Severity of depression, anxiety and stress decreased with increasing age [34], the pandemic-related psychological burden was less severe for older adults [35], age was negatively related with post-traumatic stress symptoms, as well as loneliness [36], older adults displayed less psychological distress [37], and were less psychologically vulnerable [38, 39]. Another study reported that older adults' mental wellbeing and loneliness prevalence during the COVID-19-related lockdown remained largely unchanged, based on estimates reported before the pandemic [42]. Moreover, reported mean scale scores were in several cases below the cutoff point for clinically significant symptoms [33, 35, 38-40].

With regard to studies comparing data collected prior with data collected during the pandemic, there were reports that the pandemic affected older adult's mental health, since study participants experienced during the pandemic more severe depressive, anxiety and stress symptoms [43], higher levels of loneliness [43-45] and apathy; specifically, adults younger than the age of 75 were at higher risk for developing depressive symptoms and apathy [46]. Lastly, a study confirmed the pandemic's negative psychosocial impact on older patients with multimorbidity in primary care; despite the increase in anxiety, insomnia and loneliness, depressive symptoms were unaltered [47].

Although social and emotional loneliness levels were reported to have increased during the pandemic, the effect sizes were small (Cohen's $d=0.21)$ and medium $(d=0.49)$ respectively [44]. Accordingly, overall loneliness was shown to have increased during the pandemic, the effect size was though again small $(d=0.22)$ [45]. In addition, reported mean scale scores were in several cases below the cutoff point for clinically significant anxiety, depressive symptoms [43, 46] and loneliness [44, 45, 47]. Moreover, a study reported better mental health, assessed by MHI-5, during the pandemic; the effect size was though very small $(d=0.17)$ [44].

According to baseline data from ongoing longitudinal studies, the prevalence of clinically significant depression and anxiety was low (5\%) [48]. Furthermore, there was a $19 \%$ lower risk of reporting depressive symptoms and a $22 \%$ lower risk of reporting anxiety symptoms with every 
five-year increase in age [49]. Lastly, a longitudinal study revealed that COVID-19 did not have a major impact on loneliness levels and satisfaction with life among older adults in Sweden, where a national lockdown had not been imposed [50].

Altogether, although research is ongoing, several studies suggested that older age may buffer against the COVID-19-related impact on mental health. Older people are more likely to have experienced both individual traumatic events and collective traumas, such as war, dictatorship [38], financial crises and natural disasters [59]. As such, they may be able to consider the current pandemic in a broader context, relativising its impact and show higher resilience against the COVID-19-related adverse mental health outcomes using adaptive resources $[42,53,60$, 61]. Furthermore, most studies were conducted during the acute phase of the pandemic. Therefore, results indicating worsening of older adults' mental health status during the pandemic may merely reflect a stress response that could be transient, unless proven otherwise by upcoming longitudinal studies.

On the other side, although research outcomes argue against societal ageist stereotypes depicting older adults as weak and vulnerable, four aspects should be taken into consideration: (i) the studies included in this review were conducted in developed countries. The psychological impact on older adults in least developed and landlocked developing countries [62], as well as on disadvantaged people in high-income countries, is probably underreported; (ii) sociocultural factors, such as family types and attitudes towards the elderly [63], case fatality rates, as well as duration and strictness of quarantine-related measures, are expected to differentially affect older people across different countries; (iii) a meta-analysis revealed that the psychological burden was more severe in COVID-19 patients than in the general population [64]. As such, the pandemic may bring on more adverse psychological events on COVID-19 patients, including severe ones, such as suicidal ideation [65] and may therefore have a more severe impact on older COVID-19 survivors, compared with older individuals unaffected by the disease [66]; (iv) subpopulations, such as older adults with past/current history of a mental disorder [67], with physical multimorbidity [47], residing in long-term care facilities [68] or being socially isolated [69] may be more vulnerable than older adults of the general population that participated in the reviewed studies.

This review had several limitations: (i) only one database (PubMed) was searched for relevant articles; (ii) selecting articles based on the title/abstract relevance may have resulted in omission of studies fulfilling inclusion criteria; (iii) application of strict inclusion criteria with regard to the use of validated psychometric scales and report of mean (SD) values and/or prevalence rates resulted in the exclusion of studies relevant to the review's scope; (iv) possibly relevant preprints and articles not in English were not included; (v) a thorough study quality assessment was not performed.

Conclusively, this review aimed to summarise the mental health impact of COVID-19 on older adults, based on the original research articles published in the last nine months. Researchers provided evidence that older adults may withstand the emotional strain imposed by the pandemic. Despite evidence against generalised perceptions of vulnerability, older adult's ability to adapt to adversity may be uneven, depending on cultural, social, economic and other individual factors. Taken together, the impact, moreover the long-term impact of COVID-19, is expected to vary across countries and older subpopulations and remains to be evaluated by prospective, longitudinal studies.

Author's contributions EP and VH conceived the review's scope and contributed equally, performing the article search; EP, VH, VAN and SK prepared the first draft of the manuscript. IN contributed to paper editing and critical revision of the manuscript. ID supervised the study and contributed to the final revision of the manuscript. All authors read and approved the final version of the manuscript.

Funding The authors received no financial support for the research, authorship and/or publication of this article.

Data availability Reviewed articles are accessible in PubMed.

\section{Declarations}

Conflict of interest The authors declare that they have no conflicts of interest.

Statement of human and animal rights This article does not contain any studies of human or animal subjects performed by any of the authors.

Informed consent For this type of study (comprehensive review of the literature), formal consent is not required.

\section{References}

1. Patterson KD, Pyle GF (1991) The geography and mortality of the 1918 influenza pandemic. Bull Hist Med 65:4-21

2. World Health Organization (2020) WHO Coronavirus Disease (COVID-19) Dashboard. https://covid19.who.int/?gclid=Cj0KC QiA3smABhCjARIsAKtrg6LFStnmmzbtxGzUVwRhCxyEGN aqt6-OLCfw4S2J-gkFjd200n-I91IaAiDzEALw_wcB Accessed Jan 27, 2021.

3. European Statistical System (2019). Population structure and ageing. Retrieved from https://ec.europa.eu/eurostat/statistics-expla ined/index.php/Population_structure_and_ageing

4. United Nations, Department of Economic and Social Affairs, Population Division (2019) World Population Ageing 2019: Highlights. Retrieved from https://www.un.org/en/development/ desa/population/publications/pdf/ageing/WorldPopulationAgein g2019-Highlights.pdf 
5. Hutton D and World Health Organization (2008) Older persons in emergencies: considerations for action and policy development. Retrieved from https://apps.who.int/iris/handle/10665/43817

6. Lekamwasam R, Lekamwasam S (2020) Effects of COVID-19 pandemic on health and wellbeing of older people: a comprehensive review. Ann Geriatr Med Res 24:166-172. https://doi.org/10. 4235/agmr.20.0027

7. D'cruzBanerjee MD (2020) "An invisible human rights crisis": the marginalization of older adults during the COVID-19 pandemican advocacy review. Psychiatry Res 292:113369. https://doi.org/ 10.1016/j.psychres.2020.113369

8. Grolli RE, Mingoti MED, Bertollo AG et al (2021) Impact of COVID-19 in the mental health in elderly: psychological and biological updates. Mol Neurobiol. https://doi.org/10.1007/ s12035-020-02249-x

9. Clark A, Jit M, Warren-Gash C et al (2020) Global, regional, and national estimates of the population at increased risk of severe COVID-19 due to underlying health conditions in 2020: a modelling study. Lancet Glob Health 8:e1003-e1017. https:// doi.org/10.1016/S2214-109X(20)30264-3

10. Finelli C (2020) Obesity, COVID-19 and immunotherapy: the complex relationship! Immunotherapy 12:1105-1109. https:// doi.org/10.2217/imt-2020-0178

11. Yanez ND, Weiss NS, Romand JA et al (2020) COVID-19 mortality risk for older men and women. BMC Public Health 20:1742. https://doi.org/10.1186/s12889-020-09826-8

12. Parlapani E, Holeva V, Voitsidis P et al (2020) Psychological and behavioral responses to the COVID-19 pandemic in Greece. Front Psychiatry 11:821. https://doi.org/10.3389/fpsyt.2020.00821

13. Maxfield M, Pituch KA (2020) COVID-19 worry, mental health indicators, and preparedness for future care needs across the adult lifespan. Aging Ment Health. https://doi.org/10.1080/13607863. 2020.1828272

14. Antommaria AHM, Gibb TS, McGuire AL et al (2020) Ventilator triage policies during the COVID-19 pandemic at US hospitals associated with members of the Association of Bioethics Program Directors. Ann Intern Med 173:188-194. https://doi.org/10.7326/ M20-1738

15. Popescu D, Marcoci A. (2020, April 22) Coronavirus: Allocating ICU beds and ventilators based on age is discriminatory. The Conversation. Retrieved from https://theconversation.com/coron avirus-allocating-icu-beds-and-ventilators-based-on-age-is-discr iminatory-136459

16. Prendki V, Tau N, Avni T et al (2020) A systematic review assessing the under-representation of elderly adults in COVID19 trials. BMC Geriatr 20:538. https://doi.org/10.1186/ s12877-020-01954-5

17. Pan American Health Organization (PAHO/WHO) (2020) Policy Brief: The impact of COVID-19 on older persons. Retrieved from https://www.paho.org/en/documents/policy-brief-impact-covid19-older-persons

18. Steinman MA, Perry L, Perissinotto CM (2020) Meeting the care needs of older adults isolated at home during the COVID-19 pandemic. JAMA Intern Med. https://doi.org/10.1001/jamainternmed. 2020.1661

19. Han SD, Mosqueda L (2020) Elder abuse in the COVID-19 era. JAGS. https://doi.org/10.1111/jgs.16496

20. Donovan NJ, Blazer D (2020) Social isolation and loneliness in older adults: review and commentary of a National Academies Report. Am J Geriatr Psychiatry 28:1233-1244. https://doi.org/ 10.1016/j.jagp.2020.08.005

21. Goveas JS, Shear MK (2020) Grief and the COVID-19 pandemic in older adults. Am J Geriatr Psychiatry 28:1119-1125. https:// doi.org/10.1016/j.jagp.2020.06.021

22. Lee K, Jeong GC, Yim J (2020) Consideration of the psychological and mental health of the elderly during COVID-19: a theoretical review. Int J Environ Res Public Health 17:8098. https://doi.org/10.3390/ijerph17218098

23. Flett GL, Heisel MJ (2020) Aging and feeling valued versus expendable during the COVID-19 pandemic and beyond: a review and commentary of why mattering is fundamental to the health and well-being of older adults. Int J Ment Health Addict. https:// doi.org/10.1007/s11469-020-00339-4

24. Palmer K, Monaco A, Kivipelto M et al (2020) The potential long-term impact of the COVID-19 outbreak on patients with non-communicable diseases in Europe: consequences for healthy ageing. Aging Clin Exp Res 32:1189-1194. https://doi.org/10. 1007/s40520-020-01601-4

25. Chevance A, Gourion D, Hoertel N et al (2020) Ensuring mental health care during the SARS-CoV-2 epidemic in France: a narrative review. Encephale 46:193-201. https://doi.org/10.1016/j. encep.2020.04.005

26. Banskota S, Healy M, Goldberg EM (2020) 15 Smartphone apps for older adults to use while in isolation during the COVID-19 pandemic. West J Emerg Med 21:514-525. http://escholarship. org/uc/uciem_westjem

27. Gorenko JA, Moran C, Flynn M et al (2021) Social isolation and psychological distress among older adults related to COVID-19: a narrative review of remotely-delivered interventions and recommendations. J Appl Gerontol 40:3-13. https://doi.org/10.1177/ 0733464820958550

28. Van Damme W, Van Lerberghe W (2000) Epidemics and fear. Trop Med Int Health 5:511-514. https://doi.org/10.1046/j.13653156.2000.00599.x

29. Budd J, Miller BS, Manning EM et al (2020) Digital technologies in the public-health response to COVID-19. Nat Med 26:11831192. https://doi.org/10.1038/s41591-020-1011-4

30. Strutt PA, Johnco CJ, Chen J, et al (2021) Stress and coping in older Australians during COVID-19: Health, service utilization, grandparenting, and technology use. Clin Gerontol. 1-13. https:// doi.org/10.1080/07317115.2021.1884158

31. Cabrera MA, Karamsetty L, Simpson SA (2020) Coronavirus and its implications for psychiatry: a rapid review of the early literature. Psychosomatics 61:607-615. https://doi.org/10.1016/j.psym. 2020.05.018

32. Tripathy S (2020) The COVID-19 pandemic and the elderly patient: review of current literature and knowledgebase. J Geriatr Care Res 7:79-83

33. Solomou I, Constantinidou F (2020) Prevalence and predictors of anxiety and depression symptoms during the COVID-19 pandemic and compliance with precautionary measures: age and sex matter. Int J Environ Res Public Health 17:4924. https://doi.org/ 10.3390/ijerph17144924

34. Nwachukwu I, Nkire N, Shalaby R et al (2020) COVID-19 Pandemic: age-related differences in measures of stress, anxiety and depression in Canada. Int J Environ Res Public Health 17:6366. https://doi.org/10.3390/ijerph17176366

35. Pieh C, Budimir S, Probst T (2020) The effect of age, gender, income, work, and physical activity on mental health during coronavirus disease (COVID-19) lockdown in Austria. J Psychosom Res 136:110186. https://doi.org/10.1016/j.jpsychores.2020. 110186

36. Minahan J, Falzarano F, Yazdani N et al (2020) The COVID19 pandemic and psychosocial outcomes across age through the stress and coping framework. Gerontologist gnaa205. https://doi. org/10.1093/geront/gnaa205

37. García-Portilla P, de la Fuente TL, Bobes-Bascarán T et al (2020) Are older adults also at higher psychological risk from COVID19? Aging Ment Health. https://doi.org/10.1080/13607863.2020. 1805723

38. Justo-Alonso A, García-Dantas A, González-Vázquez AI et al (2020) How did different generations cope with the COVID-19 
pandemic? Early stages of the pandemic in Spain. Psicothema 32:490-500. https://doi.org/10.7334/psicothema2020.168

39. García-Fernández L, Romero-Ferreiro V, López-Roldán PD et al (2020) Mental health in elderly Spanish people in times of COVID-19 outbreak. Am J Geriatr Psychiatry 28:1040-1045. https://doi.org/10.1016/j.jagp.2020.06.027

40. Shrira A, Hoffman Y, Bodner E et al (2020) COVID-19-related loneliness and psychiatric symptoms among older adults: the buffering role of subjective age. Am J Geriatr Psychiatry 28:12001204. https://doi.org/10.1016/j.jagp.2020.05.018

41. Wilson JM, Lee J, Shook NJ (2020) COVID-19 worries and mental health: the moderating effect of age. Aging Ment Health. https://doi.org/10.1080/13607863.2020.1856778

42. Röhr S, Reininghaus U, Riedel-Heller SG (2020) Mental wellbeing in the German old age population largely unaltered during COVID-19 lockdown: results of a representative survey. BMC Geriatr 20:489. https://doi.org/10.1186/s12877-020-01889-x

43. Krendl AC, Perry BL (2020) The impact of sheltering-in-place during the COVID-19 pandemic on older adult's social and mental well-being. J Gerontol B Psychol Sci Soc Sci. https://doi.org/10. 1093/geronb/gbaa110

44. van Tilburg TG, Steinmetz S, Stolte E et al (2020) Loneliness and mental health during the COVID-19 pandemic: a study among Dutch older adults. J Gerontol B Psychol Sci Soc Sci. https://doi. org/10.1093/geronb/gbaa111

45. Heidinger T, Richter L (2020) The effect of COVID-19 on loneliness in the elderly. An empirical comparison of pre-and peri-pandemic loneliness in community-dwelling elderly. Front Psychol. 11:585308. https://doi.org/10.3389/fpsyg.2020.585308

46. Fujita K, Inoue A, Kuzuya M et al (2021) Mental health status of the older adults in Japan during the COVID-19 pandemic. J Am Med Dir Assoc 22:220-221. https://doi.org/10.1016/j.jamda.2020. 11.023

47. Wong SYS, Zhang D, Sit RWS et al (2020) Impact of COVID19 on loneliness, mental health, and health service utilisation: a prospective cohort study of older adults with multimorbidity in primary care. Br J Gen Pract 70:e817-e824. https://doi.org/10. 3399/bjgp20X713021

48. Giebel C, Lord K, Cooper C et al (2020) A UK survey of COVID19 related social support closures and their effects on older people, people with dementia, and carers. Int J Geriatr Psychiatry. https:// doi.org/10.1002/gps.5434

49. Robb CE, de Jager CA, Ahmadi-Abhari S et al (2020) Associations of social isolation with anxiety and depression during the early COVID-19 pandemic: a survey of older adults in London, UK. Front Psychiatry 11:591120. https://doi.org/10.3389/fpsyt. 2020.591120

50. Kivi M, Hansson I, Bjälkebring P (2020) Up and about: Older adults' wellbeing during the COVID-19 pandemic in a Swedish longitudinal study. J Gerontol B Psychol Sci Soc Sci. https://doi. org/10.1093/geronb/gbaa084

51. Schweda A, Weismüller B, Bäuerle A et al (2021) Phenotyping mental health: Age, community size, and depression differently modulate COVID-19-related fear and generalized anxiety. Compr Psychiatry 104:152218. https://doi.org/10.1016/j.comppsych. 2020.152218

52. Bruine de Bruin W (2020) Age differences in COVID-19 risk perceptions and mental health: evidence from a national US survey conducted in March 2020. J Gerontol B Psychol Sci Soc Sci. https://doi.org/10.1093/geronb/gbaa074

53. Losada-Baltar A, Martínez-Huertas JÁ, Jiménez-Gonzalo L et al (2021) Longitudinal correlates of loneliness and psychological distress during the lockdown situation due to COVID-19. Effects of age and self-perceptions of aging. J Gerontol B Psychol Sci Soc Sci. https://doi.org/10.1093/geronb/gbab012
54. López J, Perez-Rojo G, Noriega C et al (2020) Psychological well-being among older adults during the COVID-19 outbreak: a comparative study of the young-old and the old-old adults. Int Psychogeriatr 32:1365-1370. https://doi.org/10.1017/S104161022 0000964

55. Davidov E, Depner F (2011) Testing for measurement equivalence of human values across online and paper-and-pencil surveys. Qual Quant 45:375-390. https://doi.org/10.1007/s11135-009-9297-9

56. Weigold A, Weigold IK, Russell EJ (2013) Examination of the equivalence of self-report survey-based paper-and-pencil and internet data collection methods. Psychol Methods 18:53-70. https://doi.org/10.1037/a0031607

57. Eysenbach G, Wyatt $\mathbf{J}$ (2002) Using the internet for surveys and health research. J Med Internet Res 4:E13. https://doi.org/10.2196/ jmir.4.2.e13

58. König R, Seifert A, Doh M (2018) Internet use among older Europeans: an analysis based on SHARE data. Univ Access Inf Soc 17:621-633. https://doi.org/10.1007/s10209-018-0609-5

59. Kolaitis G, Olff M (2017) Psychotraumatology in Greece. Eur J Psychotraumatol 8:135175. https://doi.org/10.1080/20008198. 2017.1351757

60. Kimhi S, Marciano H, Eshel Y et al (2020) Resilience and demographic characteristics predicting distress during the COVID-19 crisis. Soc Sci Med 265:113389. https://doi.org/10.1016/j.socsc imed.2020.113389

61. Fuller HR, Huseth-Zosel A (2020) Lessons in resilience: initial coping among older adults during the COVID-19 pandemic. Gerontologist. https://doi.org/10.1093/geront/gnaa170

62. United Nations (2020) World's most vulnerable countries lack the capacity to respond to a global pandemic credit: MFD/Elyas Alwazir. Retrieved from https://www.un.org/ohrlls/news/world\% E2\%80\%99s-most-vulnerable-countries-lack-capacity-respondglobal-pandemic-credit-mfdelyas-alwazir

63. Parlapani E, Holeva V, Nikopoulou VA et al (2020) Intolerance of uncertainty and loneliness in older adults during the COVID-19 pandemic. Front Psychiatry 11:842. https://doi.org/10.3389/fpsyt. 2020.00842

64. Krishnamoorthy Y, Nagarajan R et al (2020) Prevalence of psychological morbidities among general population, healthcare workers and COVID-19 patients amidst the COVID-19 pandemic: a systematic review and meta-analysis. Psychiatry Res 293:113382. https://doi.org/10.1016/j.psychres.2020.113382

65. Wang M, Hu C, Zhao Q et al (2021) Acute psychological impact on COVID-19 patients in Hubei: a multicenter observational study. Transl Psychiatry 11:133. https://doi.org/10.1038/ s41398-021-01259-0

66. Mukaetova-Ladinska EB, Kronenberg G (2020) Psychological and neuropsychiatric implications of COVID-19. Eur Arch Psychiatry Clin Neurosci. https://doi.org/10.1007/s00406-020-01210-2

67. Bobes-Bascarán T, Sáiz PA, Velasco A et al (2020) Early psychological correlates associated with COVID-19 in a Spanish older adult sample. Am J Geriatr Psychiatry 28:1287-1298. https://doi. org/10.1016/j.jagp.2020.09.005

68. Van der Roest HG, Prins M, van der Velden C et al (2020) The impact of COVID-19 measures on well-being of older long-term care facility residents in the Netherlands. J Am Med Dir Assoc. 21:1569-1570. https://doi.org/10.1016/j.jamda.2020.09.007

69. Sepúlveda-Loyola W, Rodríguez-Sánchez I, Pérez-Rodríguez $P$ et al (2020) Impact of social isolation due to COVID-19 on health in older people: Mental and physical effects and recommendations. J Nutr Health Aging. https://doi.org/10.1007/ s12603-020-1469-2

Publisher's Note Springer Nature remains neutral with regard to jurisdictional claims in published maps and institutional affiliations. 